\title{
Elevated axonal membrane permeability and its correlation with motor deficits in an animal model of multiple sclerosis
}

\author{
Gary Leung ${ }^{1}$, Melissa Tully ${ }^{2,3}$, Jonathan Tang ${ }^{1,2}$, Shengxi Wu ${ }^{4}$ and Riyi Shi ${ }^{1,2^{*}}$ (D)
}

\begin{abstract}
Background: It is increasingly clear that in addition to myelin disruption, axonal degeneration may also represent a key pathology in multiple sclerosis (MS). Hence, elucidating the mechanisms of axonal degeneration may not only enhance our understanding of the overall MS pathology, but also elucidate additional therapeutic targets. The objective of this study is assess the degree of axonal membrane disruption and its significance in motor deficits in EAE mice.

Methods: Experimental Autoimmune Encephalomyelitis was induced in mice by subcutaneous injection of myelin oligodendrocyte glycoprotein/complete Freud's adjuvant emulsion, followed by two intraperitoneal injections of pertussis toxin. Behavioral assessment was performed using a 5-point scale. Horseradish Peroxidase Exclusion test was used to quantify the disruption of axonal membrane. Polyethylene glycol was prepared as a $30 \%(\mathrm{w} / \mathrm{v})$ solution in phosphate buffered saline and injected intraperitoneally.
\end{abstract}

Results: We have found evidence of axonal membrane disruption in EAE mice when symptoms peak and to a lesser degree, in the pre-symptomatic stage of EAE mice. Furthermore, polyethylene glycol (PEG), a known membrane fusogen, significantly reduces axonal membrane disruption in EAE mice. Such PEG-mediated membrane repair was accompanied by significant amelioration of behavioral deficits, including a delay in the emergence of motor deficits, a delay of the emergence of peak symptom, and a reduction in the severity of peak symptom.

Conclusions: The current study is the first indication that axonal membrane disruption may be an important part of the pathology in EAE mice and may underlies behavioral deficits. Our study also presents the initial observation that PEG may be a therapeutic agent that can repair axolemma, arrest axonal degeneration and reduce motor deficits in EAE mice.

Keywords: Multiple sclerosis, EAE, Axonal membrane damage, Polyethylene glycol, Acrolein, Horseradish Peroxidase, Membrane permeability, Neurodegeneration

\section{Background}

Although inflammation is known to be the major pathology of multiple sclerosis (MS), the mechanisms underlying tissue damage and functional loss remain unclear $[1,2]$. While myelin degeneration has long been considered the primary neuropathological characteristic for MS, recent studies indicate that axonal

\footnotetext{
* Correspondence: riyi@purdue.edu

'Department of Basic Medical Sciences, College of Veterinary Medicine,

Purdue University, West Lafayette, IN 47907, USA

${ }^{2}$ Weldon School of Biomedical Engineering, Purdue University, West

Lafayette, IN 47907, USA

Full list of author information is available at the end of the article
}

degeneration is also an important component of the pathology [3-5]. In fact, there is strong evidence suggesting that MS is a neurodegenerative diseases [3, 6-8]. Indeed, the integrities of both myelin and axons are indispensable for neuronal function and survival [9]. Therefore, either myelin or axonal damage could theoretically lead to axonal conduction loss and degeneration seen in MS [10-13]. Consistent with this notion, it has been suggested that axonal disruption may represent irreversible neurodegeneration in patients with MS [3]. This may in part explain why conventional strategies focusing solely on myelin protection have resulted in 
few effective treatments to slow or prevent MS progression [1].

Despite its potential importance in MS, axonal damage has attracted significantly less attention compared to myelin damage while both are known to lead to neurodegeneration in MS $[6,7,14]$. Consequently, the pathological role of axonal damage in MS remains insufficiently characterized. Specifically, the key cellular processes that trigger axonal degeneration remain unclear. We have previously shown that axonal membrane damage contributes to axonal degeneration observed in CNS trauma [11-13, 15-17]. We have also shown that acrolein, a pro-inflammatory aldehyde that is capable of inflicting axonal membrane damage and functional loss [18-24], is elevated and likely plays an important pathological role in MS [25]. In light of this evidence, we speculate that damage to the axonal membrane, or axolemma, likely leads to neuronal degeneration and loss of neurological function, and therefore contributes to the development and progression of symptoms observed in MS.

Polyethylene glycol (PEG), a hydrophilic polymer, is well known for its ability to seal neuronal membranes and consequently restore integrity and associated neuronal function [13, 26-29]. In particular, it has been shown that PEG is capable of repairing axolemmal damage and provide neuroprotection in traumatic spinal cord injury [19, 26-28, 30-40]. However, the therapeutic effect of PEG has not been examined in non-traumatic CNS illnesses, such as MS, in which axonal membrane damage likely plays a role leading to axonal degeneration. Therefore, the primary focus of this study was to determine whether axolemmal disruption can be detected and to examine its possible correlation with functional deficits associated with MS. Subsequently, we also aimed to confirm the pathological role of axolemmal disruption in MS and to assess the therapeutic efficacy of administering PEG as a membrane sealant.

\section{Methods}

\section{Experimental autoimmune encephalomyelitis mice}

We certify that all applicable institutional and governmental regulations concerning the ethical use of animals were followed during the course of this research. Female C57BL/6 mice were purchased from Harlan Laboratories (Indianapolis, IN, USA) and were housed in the Purdue University veterinary animal housing facilities. Ten to twelve week old mice received two subcutaneous injections of $0.1 \mathrm{~mL}$ myelin oligodendrocyte glycoprotein/ complete Freud's adjuvant emulsion (EK-0115, Hooke Laboratories, Lawrence, MA, USA) into the upper and lower back. Immediately following the emulsion injections, $0.1 \mathrm{~mL}$ of pertussis toxin (EK-0115, Hook Laboratories) was administered intraperitoneally to the mice, and again 22-26 h later. Behavioral assessment was performed using a 5-point scale [41]. Animals were placed on a metal grate to record their walking ability and motor function. The behavioral scale used was as follows: 0 - no deficit; 1 - limp tail only; 2 - hind limb paresis without frank leg dragging; 3 - partial hind limb weakness with one or both legs dragging; 4 - complete hind limb paralysis; 5 - moribund, paralysis in hind limbs and forelimbs. These studies were approved by the Purdue Animal Care and Use Committee, Purdue University, West Lafayette, IN.

\section{Horseradish peroxidase exclusion test}

The mice were separated into 4 groups: healthy control mice, EAE mice before the onset of symptoms (presymptom), EAE mice at peak behavioral deficit (peak symptoms), and PEG-treated EAE mice. After confirmation of behavior at various pre-determined experimental end points (Fig. 1), each group of animals was anesthetized with Ketamine $(90 \mathrm{mg} / \mathrm{kg})$ and Xylazine $(10 \mathrm{mg} / \mathrm{kg}$ ) and perfused (intra-cardiac) with a cold, oxygenated Krebs solution. The spinal columns were quickly removed from the animal and a complete laminectomy was performed. The spinal cord was then excised from the vertebrae and placed in cold, oxygenated Krebs solution containing $0.015 \%$ horseradish peroxidase (Sigma

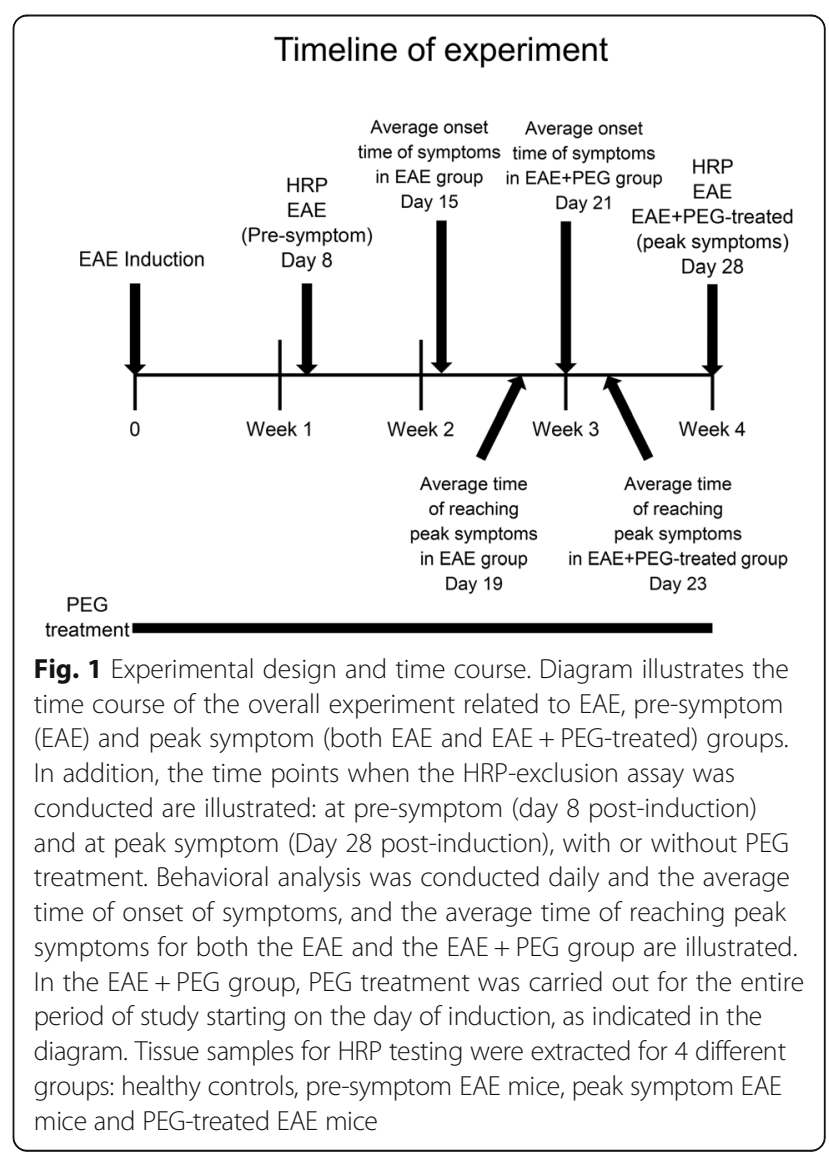


Type IV, Sigma Aldrich) for $2 \mathrm{~h}$. The tissue was then fixed in $2.5 \%$ glutaraldehyde in phosphate buffer for $4 \mathrm{~h}$ at room temperature. After fixation, a Vibratome (Electron Microscopy Science, Hatfield, PA, USA) was used to cut $30 \mu \mathrm{m}$ transverse sections of the tissue. Tissue was then processed in a diaminobenzidene solution to visualize HRP uptake by damaged. Digital images of HRPstained spinal cord sections were obtained with an optical microscope connected to a computer. Stained axons were counted and expressed as density (axons $/ \mathrm{mm}^{2}$ ) using Image $J$ analysis $[15,17,30]$. Animals were sacrificed for structural analysis at pre-induction (control), 8 days postinduction (pre-symptom), or 4 weeks post-induction (peak symptom).

\section{Polyethylene glycol treatment}

Polyethylene glycol (295906, Sigma Aldrich, St. Louis, MO, USA) was prepared as a $30 \%(\mathrm{w} / \mathrm{v})$ solution in phosphate buffered saline. The solution was then filtered for sterilization and injected into each animal every day after induction for the whole study (4 weeks postinduction). A volume of $0.1 \mathrm{~mL}$ was administered intraperitoneally daily after induction for the duration of the study. EAE only animals were administered the same amount of saline rather than PEG.

\section{Statistical analysis}

Throughout the paper, Mann Whitney $U$ test was used to compare the difference of the severity of motor defects, the onset of motor deficits, and the onset of peak symptoms between EAE and EAE + PEG-treated groups. Kruskal-Wallis test was used for comparison of membrane permeability assessed by HRP-exclusion test in various groups. The statistical significance level was set at $p<0.05$. All data are expressed as mean \pm standard error of the mean (SEM).

\section{Results}

Significant axonal membrane damage in EAE mouse and its reduction by Polyethylene glycol

Using an EAE mouse model, we first examined axonal membrane damage using a well-established HRP-exclusion assay. The integrity of axonal membrane from the spinal cord of control mice, EAE mice before the onset of symptoms (pre-symptom), and EAE mice at peak behavioral deficit (peak symptom) was examined (Fig. 1). We have found that the average HRP labeling for these conditions was $811 \pm 130$ axons $/ \mathrm{mm}^{2}, 3293 \pm 500$ axons $/ \mathrm{mm}^{2}$, and $6147 \pm 655$ axons $/ \mathrm{mm}^{2}$ respectively (Fig. 2). EAE mice at peak deficit demonstrated significantly higher levels of HRP labeling compared to control mice $(P<0.01)$. Interestingly, pre-symptom EAE mice also displayed increased axonal membrane permeability compared to control mice $(P<0.05)$.
We further examined the possibility that PEG can reduce axonal membrane permeability. Specifically, EAE mice were given daily injections of $0.1 \mathrm{~mL}$ of either polyethylene glycol $(30 \% \mathrm{w} / \mathrm{v})(\mathrm{EAE}+\mathrm{PEG})$, or saline (EAE only) beginning on the first day of induction and then daily for 4 weeks (Fig. 1). Axonal integrity based on the HRP exclusion assay was carried out at the end of the treatment period. We have found that the density of HRP-labeled axons in the EAE-PEG treated group was $1581 \pm 247$ axons $/ \mathrm{mm}^{2}$, which is significantly lower than that of the EAE only mice at peak deficit $(6147 \pm 655$ axons $/ \mathrm{mm}^{2}, p<0.01$ ) (Fig. 2).

\section{Polyethylene glycol temporally ameliorates motor deficits in EAE mice}

In addition to axonal membrane permeability examination, we also carried out behavioral analysis in two experimental groups, EAE and EAE + PEG mice. The behavioral observation for each animal was recorded daily on a 5-point scale immediately following induction and continuously for 4 weeks. The average behavioral score was calculated for each day in two groups and displayed over time in Fig. 3. The severity of behavioral deficit in the EAE/PEG-treated group was significantly lower than the EAE group during the period of day 16 to 25 days post EAE induction. When averaging the highest scores of each animal within each group, the PEG-treated EAE mice $(1.91 \pm 0.4)$ displayed a significantly lower score than the EAE mice (3.33 $\pm 0.3, P<0.05)$ (Fig. 3 upper inset). In addition, PEG treatment also significantly delayed the time of reaching peak symptoms ( $23.1 \pm 1.6$ days for EAE-PEG, and $18.7 \pm$ 0.8 days for EAE group, $P<0.05$ ) (Fig. 3 lower inset).

In addition to decreased peak symptom severity and delayed time to reach the peak symptom, PEG treated animals also showed delayed symptom onset as depicted in Fig. 4. Specifically, in the EAE group, all mice began to display their behavioral deficit between days 13 and 18. In contrast, the EAE + PEG group revealed a more dispersed result with a trend of delayed onset. Specifically, five EAE + PEG animals began exhibiting symptoms at approximately the same time as the EAE group (13-18 days induction) while the others were later in time: three exhibited no observable behavioral deficit throughout the 4week observation (counted as day 28 when averaging) while the remaining three mice showed their first motor defects at between day 20-26 post induction. Overall, the average day of onset for EAE mice that received PEG-treatment was $20.63 \pm 1.8$ days which is significantly delayed compared to EAE mice (15.42 \pm 0.4 days, $P<0.01$ ) (Fig. 4).

\section{Discussion}

Based on the current study using the HRP-exclusion assay, a well-established method of assessing axonal 


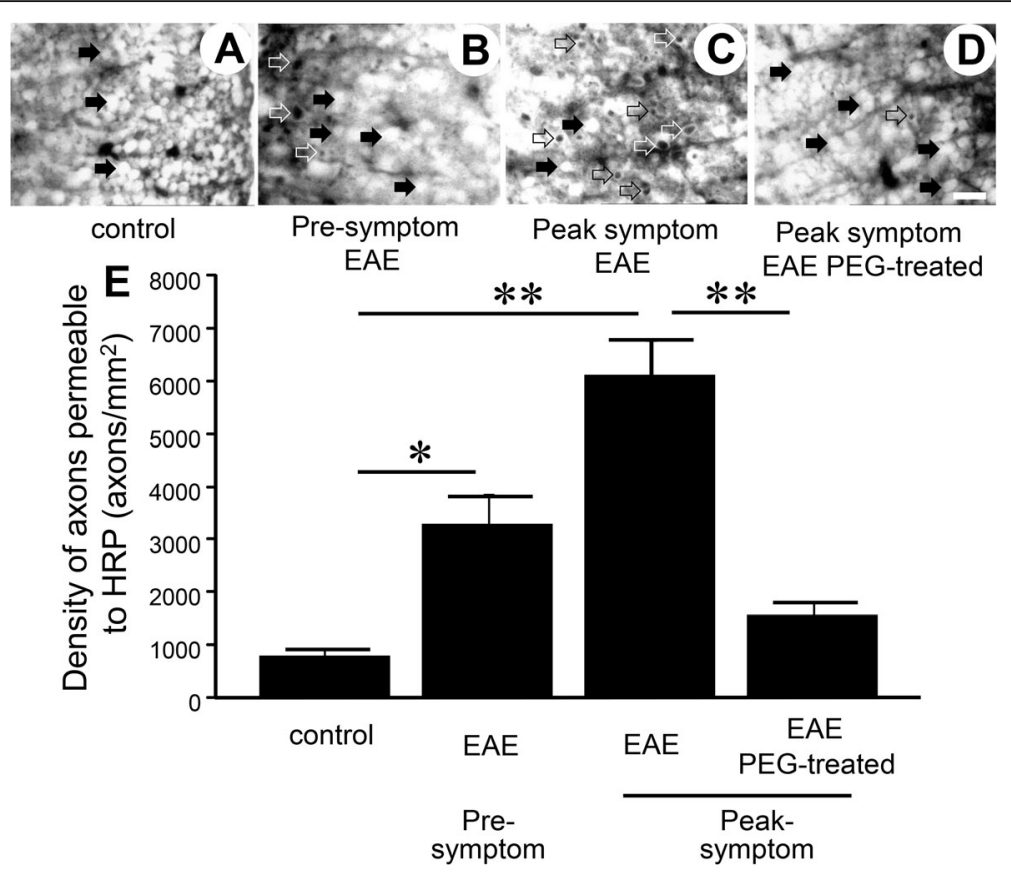

Fig. 2 Membrane permeability assessed by HRP-exclusion test. Horseradish peroxidase (HRP)-exclusion test determined amount of axonal membrane damage in healthy control mice $(n=5)$, pre-symptom EAE mice $(n=7)$, peak symptom EAE mice $(n=6)$, and peak symptom EAE + PEG-treated mice $(n=7)$. For pre-symptom EAE group, samples were taken at 8 days post-induction, before symptom emerges. The samples of peak symptom group (for both EAE and EAE + PEG-treated) were taken at 28 days post-induction. $\mathbf{a}$ - $\mathbf{d}$ The images represent HRP-stained sections of spinal cord tissue from the four groups. Solid arrows denote areas in which HRP did not penetrate the cell while the open arrows point to areas depicting HRP penetration revealing increased axonal membrane permeability. e The bar graph quantifies HRP uptake in each group. The average density for the control group was $811 \pm 130 \mathrm{axons} / \mathrm{mm}^{2}$. The peak symptom group had the highest levels of axonal damage $\left(6147 \pm 665 \mathrm{axons} / \mathrm{mm}^{2},{ }^{* *} P<0.01 \mathrm{compared}\right.$ to control) while the pre-symptom group exhibited increased levels compared to the control group (3293 $\pm 500 \mathrm{axons} / \mathrm{mm}^{2},{ }^{*} P<0.05$ compared to control). In addition, HRP labeling in the EAE/PEG-treated group $\left(1581 \pm 247 \mathrm{axons} / \mathrm{mm}^{2}\right)$ is significantly lower than EAE group $\left({ }^{* *} p<0.01\right)$. Scale bar $=10 \mathrm{~mm}$ for $\mathbf{a}, \mathbf{b}, \mathbf{c}$ and $\mathbf{d}$

membrane permeability $[11,13,15-17,30,42]$, we have determined that there is a significant level of axonal membrane disruption in EAE mice when symptoms peak. In addition, we also noted a prominent increase, although at a lesser degree, of axonal membrane damage in the pre-symptom stage in EAE mice (Fig. 2). To our knowledge, this is the first evidence of axonal membrane damage in EAE mice, an animal model MS.

Furthermore, PEG, a known membrane repairing agent [28-31], when applied daily for 4 weeks after induction, can significantly reduce axonal membrane disruption in EAE mice (Fig. 2). The restoration of axonal membrane integrity by PEG was accompanied by significant amelioration of behavioral deficits, including a delay of the onset of motor deficits typical of EAE, a delay of emergence of peak symptom, and a reduction of peak symptom severity (Figs. 3 and 4). Taken together, we have presented initial evidence that axonal membrane disruption is an important feature of the overall pathology in EAE mice that is at least in part responsible for the behavioral deficits. Our study also presents the first indication that PEG could be used as a therapeutic agent to effectively repair axolemma, arrest axonal degeneration and reduce motor deficits in EAE mice.

Although not examined in this study, it is likely that PEG-mediated axonal membrane repair also leads to the reduction of axonal degeneration, a known pathology of MS [3, 4, 43]. It is well known that axonal membrane disruption, if not repaired, will lead to axonal degeneration, neuronal cell death and overall neuronal tissue degeneration [13, 17, 44-46]. It has also been demonstrated repeatedly that PEG-mediated neuronal membrane repair can lead to the reduction of oxidative stress and mitochondrial dysfunction which are known contributors to axonal degeneration and neuronal cell death $[19,26,29,35,47]$. As such, PEG may also provide neuroprotection by indirectly suppressing oxidative stress and inflammation. Therefore, we postulate that PEGmediated membrane repair can mitigate axonal degeneration and could promote a range of cellular functions that lead to the improvement of motor function in EAE mice.

Although we did not confirm the presence of PEG inside the spinal cord in the current study following 


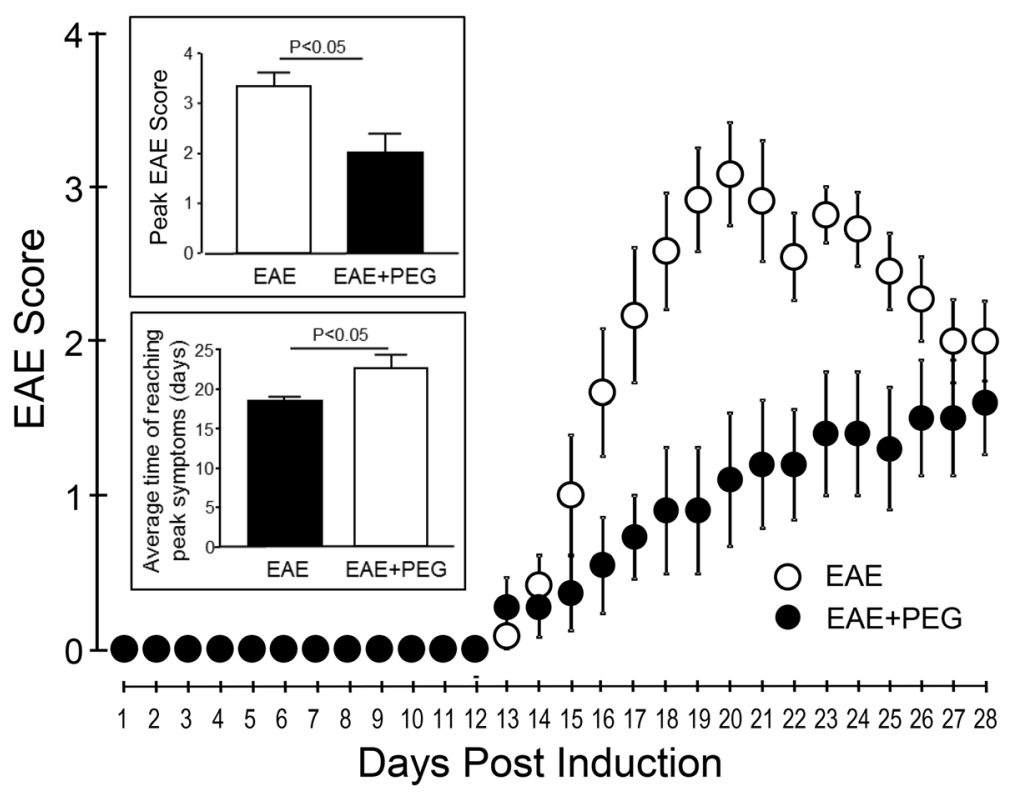

Fig. 3 PEG ameliorated the severity of motor defects and delayed the time of reaching peak motor deficits. Comparison of behavioral assessment each day between EAE (saline-treated, $n=12$ ) and EAE + PEG (EAE-treated with PEG, $n=11$ ) groups. The graph represents the average score for each group of animals throughout the study. The upper inset demonstrates that administration of PEG significantly decreased the peak symptom severity in EAE mice $(P<0.05)$. The highest score of each animal was recorded and averaged within each group to quantify the mean score of severity to be used for the upper inset. As indicated, the average of the peak EAE score in EAE + PEG group (1.91 \pm 0.4$)$ is significantly lower than that in EAE group ( $3.33 \pm 0.3, P<0.05)$. The lower inset describes the average time of reaching peak symptom of motor deficits in both $E A E$ and $E A E+P E G$ groups. For both EAE and EAE-PEG group, the time of reaching peak symptom is defined as the time that the most severe symptom, or the highest EAE score ( $\geq 2)$ first appears for each animal. If an EAE score of 2 is never reached during the experimental duration (28 days), then 28 day is used as the time of reaching peak symptom. As shown, the average time of reaching the peak EAE score in EAE + PEG group (23.1 \pm 1.6$)$ is significantly longer than that in EAE group $(18.7 \pm 0.8, P<0.05)$

systemic application, we believe the main location of PEG treatment is in the central nervous system (CNS), particularly in spinal cord. This is because we have detected significant membrane repair in spinal cord when PEG was applied comparing to no PEG (Fig. 2), and PEG is known to be able to reach spinal cord following systemic application [48]. It is unlikely that PEG-mediated neuroprotection is due to peripheral effects, considering the main pathology of EAE is in CNS [2].

Despite the strong evidence of severe axonal membrane damage in EAE, the mechanisms of such axolemmal damage remain to be elucidated. Based on the previous studies from our and other labs, we suggest that acrolein, a lipid peroxidation byproduct, may be one of the culprits. In a recent study from our lab it was shown that acrolein was increased significantly in EAE mice [25]. We have also shown that acrolein can cause membrane disruption in various preparations at levels that are likely achievable in in vivo pathological conditions [20-22, 49-51]. In fact, acrolein has been suggested to cause neuronal damage in trauma by disrupting neuronal membrane through a delayed mechanism [24, 49, 50, 52-54]. In light of this evidence, we hypothesize that acrolein may play a role in axonal membrane disruption in EAE mice. One critical piece of evidence supports this hypothesis is that hydralazine, an effective acrolein scavenger, can lower acrolein levels and reduce motor deficits in EAE mice [25]. In addition, a recent study from our group demonstrated that acrolein-mediated axonal conduction loss can be partially mitigated by a potassium channel blocker, indicating a concomitant acrolein-mediated myelin damage in addition to axonal lesions [24]. This is because augmented potassium channel activity is a known consequence of myelin damage in injured axons [55]. Consistent with such notion, we also have found that acrolein trapping treatment was associated with restoration of neuronal membrane integrity, reduced neurodegeneration and enhanced functional recovery in traumatic spinal cord injury $[21,22,24,50,51,53]$. It will be interesting to confirm the likely scenario that anti-acrolein therapy alone could lead to the preservation or restoration of axonal membrane integrity in EAE.

In the current study, in addition to the severe membrane disruption observed when symptoms peak, we also noted a less severe, yet still significant level of membrane disruption, and therefore neurodegeneration, in the presymptom period defined as a week prior to the emergence of motor deficits (Figs. 1 and 2). Therefore, significant 


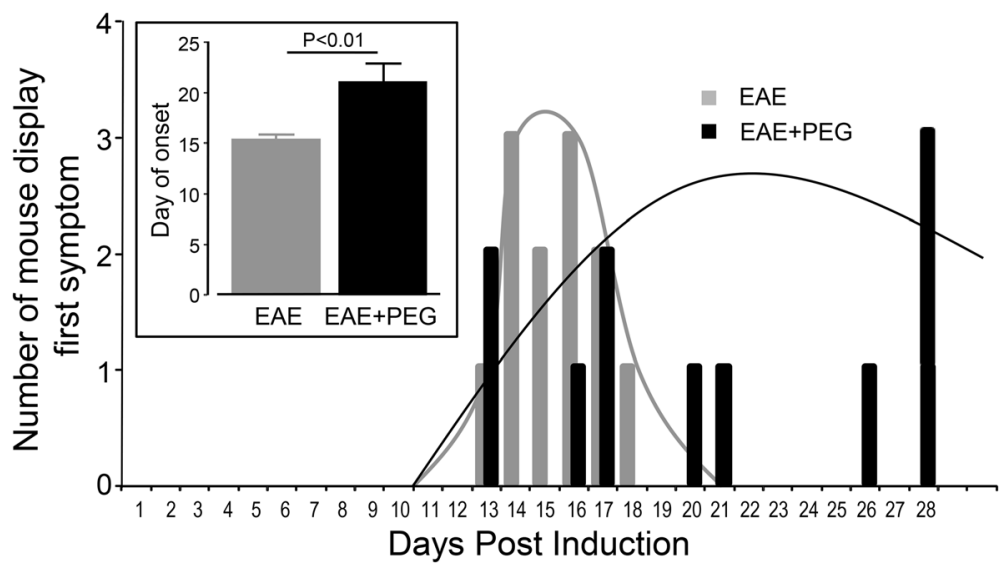

Fig. 4 PEG delayed the onset of motor deficits. Comparison of onset of symptoms between EAE (saline-treated, $n=12)$ and EAE + PEG (EAE-treated with PEG, $n=11$ ) groups. The graph represents the temporal distribution or symptom onset for EAE and EAE + PEG mice. The number of mice showing initial symptoms was recorded daily for each group. Symptom onset for EAE mice was tightly clustered early in the study while onset for EAE + PEG mice was more dispersed. The inset graph represents the average day of onset for EAE and EAE + PEG groups. Animals in EAE + PEG group developed symptoms significantly later in the study compared to EAE mice $(P<0.01)$. Three mice in the EAE + PEG-group never developed symptoms and were counted as day 28 for both graphs

level of membrane damage and neurodegeneration appear to already exist in the pre-symptom period while no concomitant noticeable behavioral deficits were detected. This phenomenon may be explained by the fact that there is a significant amount of implicit redundancy of axons to support neuronal function. Therefore, there is likely a threshold level of axonal damage and neurodegeneration that must be reached before observing behavioral changes. This would justify the notion that membrane damage could start before the onset of behavioral deficits and that the emergence of behavioral deficits signifies a critical level of axonal damage and degeneration. Hence, the initial membrane damage could theoretically serve as an indication to predict the onset of behavioral deficits at a later date.

In light of these observations related to the relationship between axonal membrane damage and behavioral performance, we suggest that axonal membrane damage in MS could serve as an important diagnostic measurement. First, it can potentially be used as a biomarker for the detection of MS before the emergence of symptoms. This could help to institute earlier treatments to deter neurodegeneration for better therapeutic outcomes provided that the pre-symptomatic axonal membrane damage can be firmly identified. Second, axonal membrane integrity could also serve as an indication of the effectiveness of membrane repair therapy such as PEG. Such a strategy is understandably dependent on development of a reliable non-invasive imaging method to detect axonal membrane disruption or degeneration.

It has become increasingly clear that, in addition to myelin damage, axonal degeneration may also play a critical role in EAE pathology. From a basic cellular biology view, the integrity of both myelin and the axonal membrane are essential for axonal conduction. Therefore, axonal degeneration along with myelin damage, are likely to be equally important contributing factors in axonal conduction failure and behavioral deficits in EAE mice $[5,12,15,17,54,56,57]$. The current study further highlighted the critical role of axonal damage, and axolemmal disruption in particular, in neurodegeneration and the functional loss in MS. To further stress the importance of axonal degeneration in MS, there is evidence that axonal damage and neurodegeneration may be the main cause of functional loss without obvious myelin damage in some human case of MS. For example, based on a histopathologic investigation using autopsy tissue from MS patients, Trapp and his colleagues have shown compelling evidence of axonal degeneration in the absence of myelin loss [43]. Therefore, axonal damage, a key feature of neurodegeneration, may not just be one of the important compounding pathological factors, but rather it may be among the primary and critical factors that are sufficient to cause clinical functional deficits in MS. To further highlight the importance and causal role of axonal damage in MS pathology, we have noted a significantly higher susceptibility of acrolein-mediated axonal damage compared to acrolein-mediated myelin damage. Specifically, Shi and his colleagues have found that using an ex vivo preparation of extracted rodent spinal cord segment, the threshold of acrolein needed to inflict membrane damage is two magnitudes lower than that needed to cause significant myelin damage [10, 49]. This suggests that in MS patients, axonal degeneration may be, in some cases, the primary pathology that precedes myelin damage. Furthermore, due to the existing 
evidence of damaging both axon and myelin, acrolein may be a critical link for the damage of both myelin and axons, two known pathologies in EAE. This hypothesis is supported by the factor that acrolein scavenging could mitigate the damage of both myelin and axons [25, 26, 29].

The emergence of the importance of axonal damage as the critical pathology warrants closer examination of our existing therapeutic strategies as well as our efforts to establish new therapies. It appears reasonable to suggest that a treatment regimen should include axonal repair and protection in addition to myelin protection. This combination of treatments may be a synergistic treatment strategy and could result in increased efficacy. We have previously shown that both anti-acrolein (by hydralazine) and membrane repair (by PEG), when used alone, can offer significant, albeit partial alleviation of behavioral deficits [25] (Fig. 3). Furthermore, though PEG significantly delayed the onset and reduced severity in most of the symptomatic periods, such symptom reduction was temporary. PEG treatment did not lead to significant behavioral improvement beyond 26 days post induction, despite effective sealing of the axonal membrane. Therefore, it appears that PEG-mediated membrane repair alone cannot offer long-term symptom reduction. This could be the case for at least two reasons. First, this may indicate that membrane repair strategies need to be combined with approaches that remove causes of cellular damage, such as acrolein, to ultimately protect the cell. Second, although a proven axonal membrane repair agent, PEG has not been shown to also repair myelin damage.

\section{Conclusions}

Our findings demonstrate that there is significant axonal membrane damage in addition to myelin destruction and both likley contribute to neurodegeneration in EAE mice. Further, the impairment of axon and myelin may require distinct protective measures and both are critical for a normalization of neuronal function. Taken together, PEG-mediated membrane repair strategy may need to be combined with other measures designed to protect and repair myelin in order to maximize the therapeutic effect and ultimate functional preservation and recovery in MS.

\section{Abbreviations}

EAE: Experimental autoimmune encephalomyelitis; MS: Multiple sclerosis; PEG: Polyethylene glycol

\section{Acknowledgements}

Not applicable.

\section{Funding}

This work was supported by the State of Indiana and the Indiana Clinical and Translational Sciences Institute (PHS NCCR \# TL1RR025759 and \# RR025761).

\section{Availability of data and materials}

All data generated or analyzed during this study are included in this published article.

\section{Authors' contributions}

GL, MT and RS conceived the experiments, GL, MT and JT, conducted the experiments, GL, MT, JT, SW and RS analyzed the results, all authors have read and approved the final manuscript.

\section{Competing interests}

Riyi Shi is the co-founder of Neuro Vigor, a start-up company with business interests of developing effective therapies for CNS neurodegenerative diseases and trauma. The authors declare that they have no competing interests.

Consent for publication

Not applicable.

\section{Ethics approval and consent to participate}

Mouse studies were conducted in accordance with guidelines mandated by the Purdue Animal Care and Use Committee (PACUC) at Purdue University, West Lafayette, IN, USA. The current study was specifically approved by PACUC of Purdue University (protocol \# 11110002876).

\section{Author details}

'Department of Basic Medical Sciences, College of Veterinary Medicine, Purdue University, West Lafayette, IN 47907, USA. Weldon School of Biomedical Engineering, Purdue University, West Lafayette, IN 47907, USA. ${ }^{3}$ MSTP program, Indiana University School of Medicine, Indianapolis, IN, USA. ${ }^{4}$ Department of Neurobiology, Fourth Military Medical University, Xi'an, China.

Received: 11 January 2017 Accepted: 16 February 2017

Published online: 28 February 2017

\section{References}

1. Compston A, Coles A. Multiple sclerosis. Lancet. 2008:372:1502-17.

2. Gold R, Linington C, Lassmann H. Understanding pathogenesis and therapy of multiple sclerosis via animal models: 70 years of merits and culprits in experimental autoimmune encephalomyelitis research. Brain. 2006;129:1953-71.

3. Trapp BD, Nave KA. Multiple sclerosis: an immune or neurodegenerative disorder? Annu Rev Neurosci. 2008:31:247-69.

4. Dutta R, Trapp BD. Pathogenesis of axonal and neuronal damage in multiple sclerosis. Neurology. 2007:68:S22-31. discussion S43-54.

5. Tully M, Shi R. New insights in the pathogenesis of multiple sclerosis-role of acrolein in neuronal and myelin damage. Int J Mol Sci. 2013;14:20037-47.

6. Stadelmann C. Multiple sclerosis as a neurodegenerative disease: pathology, mechanisms and therapeutic implications. Curr Opin Neurol. 2011:24:224-9.

7. Frischer JM, Bramow S, Dal-Bianco A, Lucchinetti CF, Rauschka H, Schmidbauer $\mathrm{M}$, Laursen $\mathrm{H}$, Sorensen $\mathrm{PS}$, Lassmann $\mathrm{H}$. The relation between inflammation and neurodegeneration in multiple sclerosis brains. Brain. 2009;132:1175-89.

8. Haider L, Simeonidou C, Steinberger G, Hametner S, Grigoriadis N, Deretzi G, Kovacs GG, Kutzelnigg A, Lassmann H, Frischer JM. Multiple sclerosis deep grey matter: the relation between demyelination, neurodegeneration, inflammation and iron. J Neurol Neurosurg Psychiatry. 2014;85:1386-95.

9. Kandel ER, Schwartz JH, Jessell TM. Principles of neural science. 4th ed. New York: McGraw-cbrhill; 2000.

10. Shi Y, Sun W, McBride JJ, Cheng JX, Shi R. Acrolein induces myelin damage in mammalian spinal cord. J Neurochem. 2011:117:554-64.

11. Shi R, Whitebone J. Conduction deficits and membrane disruption of spinal cord axons as a function of magnitude and rate of strain. J Neurophysiol. 2006;95:3384-90.

12. Ouyang H, Sun W, Fu Y, Li J, Cheng JX, Nauman E, Shi R. Compression induces acute demyelination and potassium channel exposure in spinal cord. J Neurotrauma. 2010;27:1109-20.

13. Hendricks BK, Shi R. Mechanisms of neuronal membrane sealing following mechanical trauma. Neurosci Bull. 2014;30:627-44.

14. Charil A, Filippi M. Inflammatory demyelination and neurodegeneration in early multiple sclerosis. J Neurol Sci. 2007;259:7-15.

15. Shi R. The dynamics of axolemmal disruption in guinea pig spinal cord following compression. J Neurocytol. 2004;33:203-11. 
16. Shi R, Pryor JD. Pathological changes of isolated spinal cord axons in response to mechanical stretch. Neuroscience. 2002;110:765-77.

17. Shi R, Asano T, Vining NC, Blight AR. Control of membrane sealing in injured mammalian spinal cord axons. J Neurophysiol. 2000;84:1763-9.

18. Shi R, Luo J, Peasley MA. Acrolein inflicts axonal membrane disruption and conduction loss in isolated guinea pig spinal cord. Neuroscience. 2002;115 337-40.

19. Luo J, Shi R. Diffusive oxidative stress following acute spinal cord injury in guinea pigs and its inhibition by polyethylene glycol. Neurosci Lett. 2004; 359:167-70.

20. Liu-Snyder P, McNally H, Shi R, Borgens RB. Acrolein-mediated mechanisms of neuronal death. J Neurosci Res. 2006;84:209-18.

21. Hamann K, Durkes A, Ouyang H, Uchida K, Pond A, Shi R. Critical role of acrolein in secondary injury following ex vivo spinal cord trauma. J Neurochem. 2008;107:712-21.

22. Hamann K, Nehrt G, Ouyang H, Duerstock B, Shi R. Hydralazine inhibits compression and acrolein-mediated injuries in ex vivo spinal cord. J Neurochem. 2008;104:708-18.

23. Shi R, Rickett $T$, Sun W. Acrolein-mediated injury in nervous system trauma and diseases. Mol Nutr Food Res. 2011;55:1320-31.

24. Yan R, Page JC, Shi R. Acrolein-mediated conduction loss is partially restored by K+ channel blockers. J Neurophysiol. 2016;115:701-10.

25. Leung G, Sun W, Zheng L, Brookes S, Tully M, Shi R. Anti-acrolein treatment improves behavioral outcome and alleviates myelin damage in experimental autoimmune enchephalomyelitis mouse. Neuroscience. 2011;173:150-5.

26. Luo J, Borgens R, Shi R. Polyethylene glycol immediately repairs neuronal membranes and inhibits free radical production after acute spinal cord injury. J Neurochem. 2002;83:471-80.

27. Luo J, Borgens R, Shi R. Polyethylene glycol improves function and reduces oxidative stress in synaptosomal preparations following spinal cord injury. J Neurotrauma. 2004;21:994-1007.

28. Shi R, Borgens RB. Acute repair of crushed guinea pig spinal cord by polyethylene glycol. J Neurophysiol. 1999;81:2406-14.

29. Nehrt A, Hamann K, Ouyang H, Shi R. Polyethylene glycol enhances axolemmal resealing following transection in cultured cells and in ex vivo spinal cord. J Neurotrauma. 2010;27:151-61.

30. Shi R, Borgens RB. Anatomical repair of nerve membranes in crushed mammalian spinal cord with polyethylene glycol. J Neurocytol. 2000;29:633-43.

31. Borgens RB, Shi R. Immediate recovery from spinal cord injury through molecular repair of nerve membranes with polyethylene glycol. Faseb J. 2000;14:27-35.

32. Shi R. Polyethylene glycol repairs membrane damage and enhances functional recovery: a tissue engineering approach to spinal cord injury. Neurosci Bull. 2013;29:460-6.

33. Shi $Y$, Kim S, Huff TB, Borgens RB, Park K, Shi R, Cheng JX. Effective repair of traumatically injured spinal cord by nanoscale block copolymer micelles. Nat Nanotechnol. 2010;5:80-7.

34. Baptiste DC, Austin JW, Zhao W, Nahirny A, Sugita S, Fehlings MG. Systemic polyethylene glycol promotes neurological recovery and tissue sparing in rats after cervical spinal cord injury. J Neuropathol Exp Neurol. 2009;68:661-76.

35. Luo J, Shi R. Polyethylene glycol inhibits apoptotic cell death following traumatic spinal cord injury. Brain Res. 2007;1155:10-6.

36. Liu-Snyder P, Logan MP, Shi R, Smith DT, Borgens RB. Neuroprotection from secondary injury by polyethylene glycol requires its internalization. J Exp Biol. 2007;210:1455-62.

37. Ditor DS, John SM, Roy J, Marx JC, Kittmer C, Weaver LC. Effects of polyethylene glycol and magnesium sulfate administration on clinically relevant neurological outcomes after spinal cord injury in the rat. J Neurosci Res. 2007;85:1458-67.

38. Detloff MR, Lavik E, Fisher LC, Langer R, Basso DM. Polyethylene glycol administration after moderate spinal cord injury decreases lesionsize and improves locomotor recovery. J Neurotrauma. 2005;22:1219.

39. Duerstock BS, Borgens RB. Three-dimentional morphometry of spinal cord injury follow polyethylene glycol treatment. J Exp Biol. 2002;205:13-24.

40. Borgens RB, Shi R, Bohnert D. Behavioral recovery from spinal cord injury following delayed application of polyethylene glycol. J Exp Biol. 2002;205:1-12

41. Kalyvas A, David S. Cytosolic phospholipase A2 plays a key role in the pathogenesis of multiple sclerosis-like disease. Neuron. 2004;41:323-35.

42. Shi R, Pryor JD. Temperature dependence of membrane sealing following transection in mammalian spinal cord axons. Neuroscince. 2000;98:157-66.
43. Bjartmar C, Kinkel RP, Kidd G, Rudick RA, Trapp BD. Axonal loss in normalappearing white matter in a patient with acute MS. Neurology. 2001;57: 1248-52.

44. Schanne FAX, Kane AB, Young EE, Farber JL. Calcium dependence of toxic cell death: a final common pathway. Science. 1979;206:700-2.

45. Xie X, Barrett JN. Membrane resealing in cultured rat septal neurons after neurite transection: evidence for enhancement by Ca2 + -triggered protease activity and cytoskeletal disassembly. J Neurosci. 1991;11:3257-67.

46. Schlaepfer WW, Bunge RP. Effects of calcium ion concentration on the degeneration of amputated axons in tissue culture. J Cell Biol. 1973;59:456-70.

47. Chen H, Quick E, Leung G, Hamann K, Fu Y, Cheng JX, Shi R. Polyethylene glycol protects injured neuronal mitochondria. Pathobiology. 2009;76:117-28.

48. Borgens RB, Bohnert D. Rapid recovery from spinal cord injury following subcutaneously administered polyethylene glycol. J Neurosci Res. 2001;66: 1179-86.

49. Luo J, Shi R. Acrolein induces axolemmal disruption, oxidative stress, and mitochondrial impairment in spinal cord tissue. Neurochem Int. 2004;44: 475-86.

50. Hamann K, Shi R. Acrolein scavenging: a potential novel mechanism of attenuating oxidative stress following spinal cord injury. J Neurochem. 2009;111:1348-56.

51. Liu-Snyder $P$, Borgens RB, Shi R. Hydralazine rescues PC12 cells from acrolein-mediated death. J Neurosci Res. 2006;84:219-27.

52. Shi R, Luo L. The role of acrolein in spinal cord injury. Appl Neurol. 2006;2:22-7

53. Park J, Zheng L, Marquis A, Walls M, Duerstock B, Pond A, Vega-Alvarez S, Wang $\mathrm{H}$, Ouyang Z, Shi R. Neuroprotective role of hydralazine in rat spinal cord injury-attenuation of acrolein-mediated damage. J Neurochem. 2014; 129:339-49.

54. Park J, Muratori B, Shi R. Acrolein as a novel therapeutic target for motor and sensory deficits in spinal cord injury. Neural Regen Res. 2014;9:677-83.

55. Shi R, Sun W. Potassium channel blockers as an effective treatment to restore impulse conduction in injured axons. Neurosci Bull. 2011;27:36-44.

56. Leung G, Sun W, Brookes S, Smith D, Shi R. Potassium channel blocker, 4-Aminopyridine-3-Methanol, restores axonal conduction in spinal cord of an animal model of multiple sclerosis. Exp Neurol. 2011;227:232-5.

57. Shi R, Page JC, Tully M. Molecular mechanisms of acrolein-mediated myelin destruction in CNS trauma and disease. Free Radic Res. 2015;49:888-95.

\section{Submit your next manuscript to BioMed Central and we will help you at every step:}

- We accept pre-submission inquiries

- Our selector tool helps you to find the most relevant journal

- We provide round the clock customer support

- Convenient online submission

- Thorough peer review

- Inclusion in PubMed and all major indexing services

- Maximum visibility for your research

Submit your manuscript at www.biomedcentral.com/submit 